\title{
Combustion of Submillimeter Heptane/Methanol and Heptane/Ethanol Droplets in Reduced Gravity
}

\author{
I. Aharon, ${ }^{1,2}$ V. K. Tam, ${ }^{1,3}$ and B. D. Shaw ${ }^{1}$ \\ ${ }^{1}$ Mechanical and Aerospace Engineering Department, University of California, Davis, CA 95616, USA \\ ${ }^{2}$ Rafael, Haifa 31021, Israel \\ ${ }^{3}$ Tesla Motors, 3500 Deer Creek Road, Palo Alto, CA 94304, USA
}

Correspondence should be addressed to B. D. Shaw; bdshaw@ucdavis.edu

Received 29 September 2012; Revised 8 January 2013; Accepted 8 January 2013

Academic Editor: Constantine D. Rakopoulos

Copyright (C) 2013 I. Aharon et al. This is an open access article distributed under the Creative Commons Attribution License, which permits unrestricted use, distribution, and reproduction in any medium, provided the original work is properly cited.

\begin{abstract}
Reduced-gravity experiments were performed on combustion of droplets composed of $\mathrm{n}$-heptane mixed with methanol or ethanol. The initial alcohol mass fraction in a droplet was $0 \%$ (pure heptane) or $5 \%$. The experiments were performed at $0.1 \mathrm{MPa}$ and $25^{\circ} \mathrm{C}$ with air or with ambients of oxygen and helium with oxygen mole fractions of 0.3 or 0.4. Initial droplet diameters were in the range $0.67 \mathrm{~mm}$ to $0.92 \mathrm{~mm}$. After considering measurement uncertainties, burning rates decreased appreciably as the initial droplet diameter increased for combustion in air but not for combustion in the oxygen/helium environments. It was also found that addition of either methanol or ethanol did not influence burning rates appreciably and that burning rates were larger for the oxygen/helium environments than for air if initial droplet diameter dependences were accounted for.
\end{abstract}

\section{Introduction}

Because global petroleum reserves are limited, there is a need to develop alternative fuels. Alcohols are considered to be promising for this purpose. For example, blends of methanol and ethanol with hydrocarbon fuels have been suggested as a possible approach to extending the availability of liquid petroleum-based fuels and also for reducing soot emissions. Blending of ethanol with hydrocarbons is presently employed in practice, for example, with oxygenated gasoline. It is thus of interest to study combustion droplets composed of blends of hydrocarbons with alcohols.

There are numerous experimental methods available for studying the combustion characteristics of fuels. These methods range from experiments in practical devices such as engines, which can be difficult to control and interpret at a detailed level, to fundamental experiments that allow one to focus on specific details of the combustion under wellcontrolled conditions. In the present case, we are interested in combustion of liquid fuels. Because liquid fuels are typically burned as sprays, many researchers have focused on studying the behaviors of individual droplets, which is the methodology we pursue here. In particular, we employ reduced gravity so that the complicating effects of buoyancy are strongly reduced. We also study droplets in the submillimeter size range, which can be observed in detail. It is much more difficult to acquire useful data for the smaller droplets $(\mathrm{O}(10-$ $100 \mu \mathrm{m})$ ) that are generally present in practical sprays.

The study of single droplet combustion is useful on both scientific and practical grounds. Studying individual droplets may enable the development of droplet submodels for computational models of sprays and increase the understanding of the basic physics of droplet vaporization and combustion, for example, for comparison with detailed models that can only be developed for simplified situations such as for an individual droplet. If models and experiments agree satisfactorily for simplified situations, then predictions for complex situations such as sprays, where there are generally limited experimental data, can be made with more confidence.

In the present paper, results from experiments on reduced-gravity combustion of droplets consisting of heptane, heptane/methanol, or heptane/ethanol mixtures are 


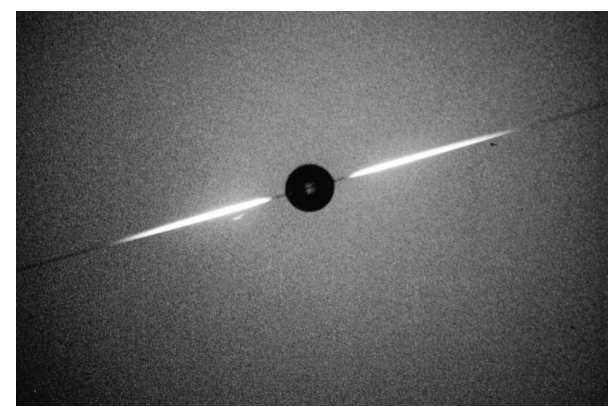

Figure 1: Digitized frame of $16 \mathrm{~mm}$ film from the droplet view camera.

described, with a particular focus on investigating droplet burning rates. The initial alcohol mass fraction in a droplet, $Y$, was set to be 0 or 0.05 . All experiments were performed at atmospheric pressure $(0.1 \mathrm{MPa})$. The environmental gas composition was atmospheric air or oxygen/helium mixtures with molar oxygen percentages, $X$, of $30 \%$ or $40 \%$. The initial droplet diameters investigated were in the range $0.67-0.92 \mathrm{~mm}$. The droplets were supported on thin $(14 \mu \mathrm{m}$ diameter) silica fibers. In addition, all experiments were conducted in an environment that was about $25^{\circ} \mathrm{C}$. While there have been numerous investigations of reduced-gravity droplet combustion, to the best of our knowledge the only hydrocarbon/alcohol mixture droplets burned in reduced gravity were toluene/methanol mixtures and mixtures of hexanol with nonane or jet fuel [1-3].

\section{Experimental Apparatus}

Experiments were performed using the 2.2 Second Drop Tower at the NASA Lewis Research Center in Cleveland, $\mathrm{OH}$, USA. This facility provides microgravity levels of about $10^{-4}$ of normal earth gravity [4]. An experiment involves deployment of a droplet of about $1 \mathrm{~mm}$ diameter or less with minimal velocity relative to the surrounding gas. Fluid is dispensed by solenoid-activated step syringes and a liquid bridge is formed between opposed needle tips. The droplet formed is slowly stretched over a small distance by retracting the needles. This stretching minimizes droplet perturbations and drift velocities when droplets are deployed. At deployment, the needles are symmetrically and rapidly retracted and a nearly stationary droplet is left behind. To deploy stationary droplets, the droplets were generated around a $14 \mu \mathrm{m}$ diameter silica fiber.

Ignition via two symmetrically spaced ignition sparks occurred about $60 \mathrm{~ms}$ after deployment. The spark current duration was about $700 \mu$ s and the energy of each spark was about $250 \mathrm{~mJ}$. Following ignition, the spark electrodes were retracted away from the burning droplet. The deployment and ignition systems were contained within a combustion chamber. Two high-speed $16 \mathrm{~mm}$ Milliken cameras were employed to capture the droplet and flame images. The droplet view was backlit, while the flame view was not. The droplet and flame view cameras operated at 375 and 125 frame/s, respectively. The flame and droplet images

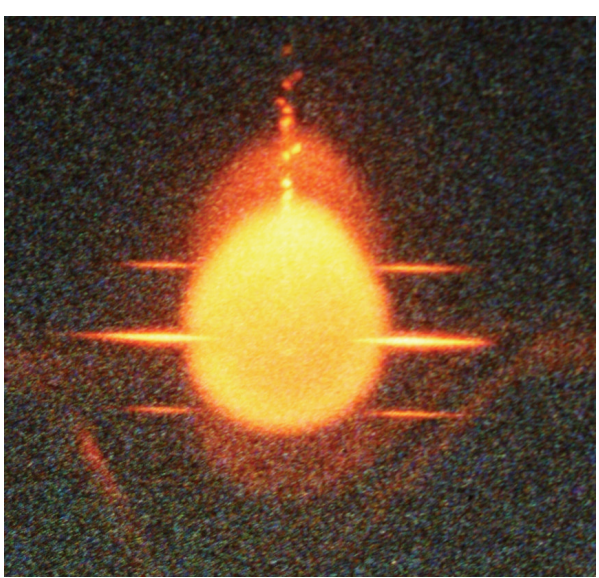

FIgure 2: Digitized frame of $16 \mathrm{~mm}$ film from the flame view camera.

were also captured simultaneously with CCD video cameras. More detailed descriptions of the apparatus may be found elsewhere [5].

\section{Data Analysis Methods}

The data obtained were in the form of video and cine images. These images were digitized and analyzed using computer-based methods. For digitization of images from the cine films, a film transport system (FTS) from Mekel Engineering Inc. [6] was employed. This FTS was employed in an earlier cine film digitization and analysis system used by NASA [7], though this earlier system did not employ a high-resolution color camera for digitization. The FTS allows precise control of film movement over an illumination source at a controllable speed. To capture a frame of film, a Canon EOS 20D digital camera (3504 by 2236 pixels) was used with close-up lenses. MATLAB and the Instrument Control Toolbox [8] were used to control the FTS with ASCII commands.

Figure 1 shows a representative digitized frame of film from the droplet view camera at $1 \mathrm{x}$ life-size magnification. The droplet, which is supported by a thin fiber, is clearly seen in detail even without further magnification. Figure 2 provides an example of a digitized frame of film from the flame view camera. It is noted that multiple images of the flame appear in this frame. These multiple images were a result of reflections that occurred with a window in the pressure vessel that contained the burning droplets. The reflected images were of lower intensity and were easily accounted for during the analysis. Reflections were accounted for in the flame images by employing a prescribed threshold.

Digitized images were processed and analyzed on a computer using MATLAB and the Image Processing Toolbox $[9,10]$. The image analysis code is based on a scheme where individual JPEG images are analyzed in sequential order. To begin, each image requires rotation as the camera originally recorded the footage at an odd angle as evidenced from the angled fiber seen in Figure 1. After the rotation to orient the fiber horizontally, each image is cropped to a specified region 


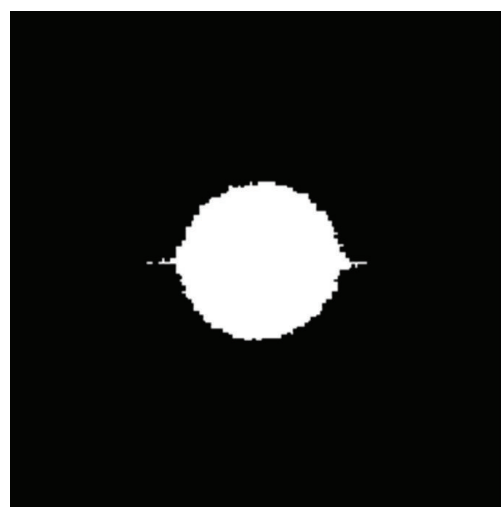

(a)

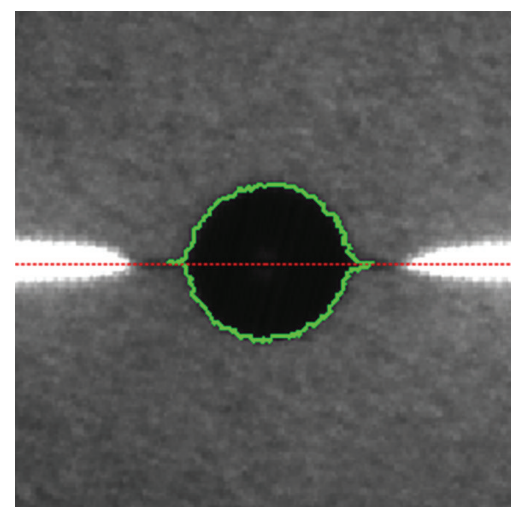

(b)

FIGURE 3: Images showing (a) the binary image with a thin fiber and (b) the droplet outline and fiber location.

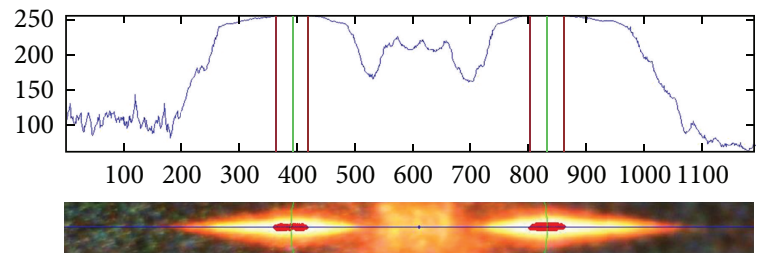

(a)

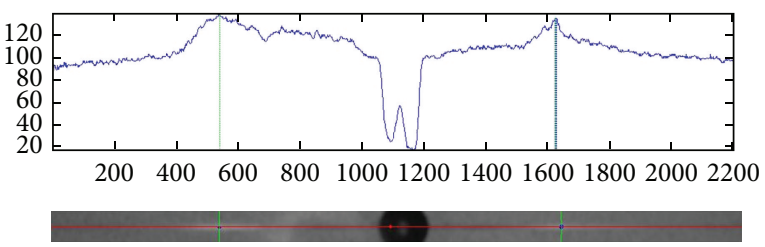

(b)

FIGURE 4: Plots of the brightest pixel with the corresponding region of interest for (a) the flame view camera and (b) the droplet view camera.

of interest, focusing on just the droplet and fiber. A threshold [10] is calculated using Otsu's method [11] to convert the image into a black and white binary as shown in Figure 3(a), with a white droplet over a black background. Otsu's method selects a threshold that minimizes the within-class variance or maximizes the between-class variance of the image gray-level histogram. The resulting binary image allows differentiation of the droplet from the background.

Because a backlit configuration was used, a bright spot is sometimes observed at the center of the droplet. The fiber inside the droplet is also frequently seen in the bright spot. The nonuniform intensity inside the droplet may result in a binary image that shows a hole inside of the droplet. Therefore, all holes within the droplet object are filled in to accurately represent the entire droplet.

The droplet is located in the binary image by selecting the largest object that is present. Its outline is then traced to retrieve the droplet boundary coordinates. After retrieval of these coordinates, the fiber is located by selecting the widest row of white pixels on the droplet. The assumption is that the widest row will indicate where the droplet interfaces with the fiber. Figure 3(b) shows the droplet outline and fiber location with a solid line tracing the droplet and a dotted line tracing the fiber.

Using the binary image, the number of pixels composing the droplet is determined, which provides the projected area of the droplet. From the projected area, the droplet diameter is obtained under the assumption that the droplet is spherical. Also, since the fiber is thin, it is not present in the binary image to a significant degree and its effect on the droplet area is negligible for these experiments.

Tracking the diameter of the flame is also important, for example, to obtain the flame-to-droplet diameter ratio. The edge of the flame is sometimes observed in droplet combustion experiments as a blue circular outline. However, the blue region is subtle and it can be difficult to observe despite the use of color photography. Furthermore, a more intense yellow portion of the flame caused by soot often renders the blue edge of flame even more difficult to see. One way to track the flame is to observe where it makes contact with the fiber. The portion of the fiber in contact with the hottest part of the flame, which should approximately correspond to the center of the blue zone, will glow the brightest.

By tracking the glowing portions of the fiber, the diameter of the flame is obtained by measuring the distance between the most intense glowing points along the fiber. First, a thin rectangular region of interest is selected to include the two glowing sections of the fiber. The region of interest is then remapped from a color image into a grayscale intensity image. Scanning across the columns of the intensity image, the values of the pixels of maximum intensity in each column are found and plotted. Figure 4(a) contains an example of a selected region of interest and the resulting plot of the brightest pixel 


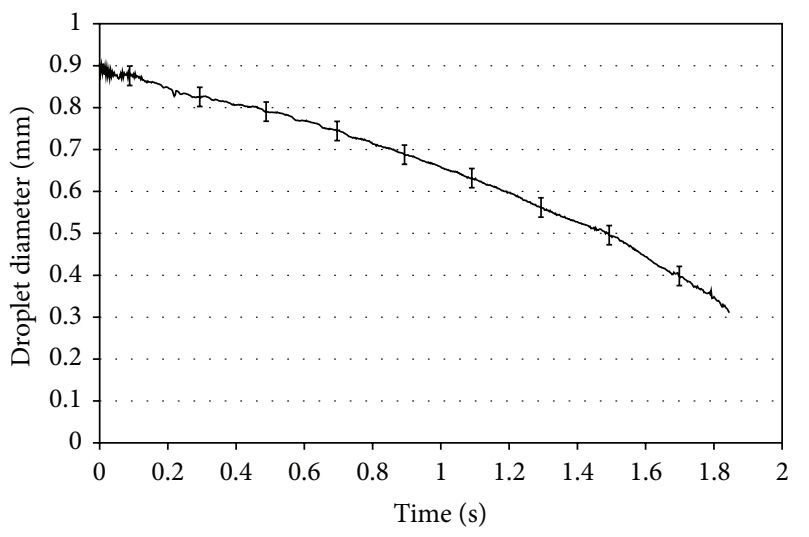

(a)

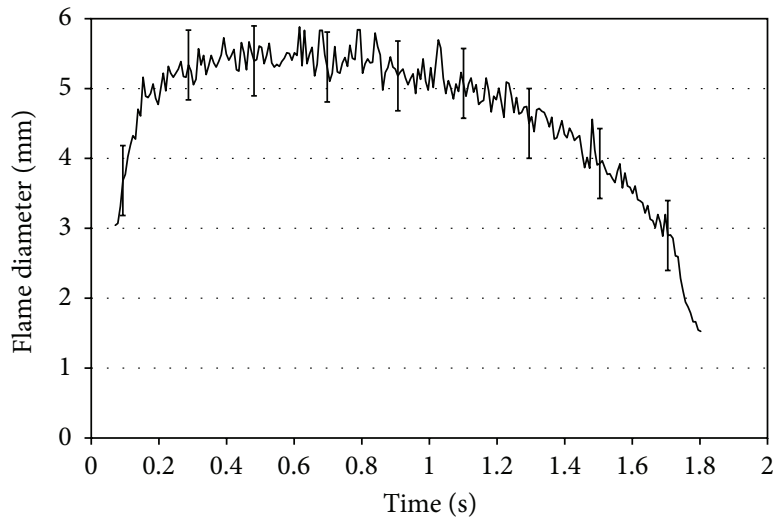

(b)

FIGURE 5: Representative plots of (a) droplet diameter and (b) flame diameter histories for a heptane/methanol droplet in air.

in each column for an image from the flame view camera. Figure 4(b) shows a corresponding image from the droplet view camera. The plots show where the brightest pixels in the image are located. In some cases, multiple points are observed to have the same maximum intensity and an average is taken to define the location of the edge of the flame. Figure 4(a) shows many pixels with the maximum intensity value. These points are highlighted with dots in the region of interest image. Taking the difference between the edge of the flame in the left and right glowing sections of the fiber gives the flame diameter. The center of the flame is also recorded and used to adaptively move the region of interest and also to track movement of the flame.

It is noted that Choi et al. [12] developed a computerbased image processing system for analysis of droplet images from cine films. However, the camera they used was of significantly lower resolution than what was used here. Choi et al. [12] also did not consider fiber-supported droplets or flame tracking.

\section{Results and Discussion}

Ignition as visualized from the flame view was associated with the appearance of color combinations (green, yellow, and blue). The blue coloring is chemiluminescence from electronically excited $\mathrm{CH}$ radicals, while the yellow coloring is broadband radiation from soot. The green coloring, which was likely from $\mathrm{C}_{2}$ molecules, quickly decayed until it could not be imaged. The flames of the heptane and heptane/methanol mixture droplets in air and the heptane/ethanol mixture droplet in $\mathrm{O}_{2} / \mathrm{He}(X=0.4)$ (after ignition) appeared as yellow spheres. The blue region, which marks the primary reaction zone, was not visible with these mixtures because of the intense soot radiation. Visual observations suggested that the yellow color intensity was spatially and temporally nonuniform. In contrast, the heptane/ethanol mixture droplet burning in $\mathrm{O}_{2} / \mathrm{He}(X=0.30)$ displayed a blue spherical flame throughout almost the entire test except when soot was present during the initial stages. The absence of soot in this case would have been the result of the lower flame temperature at $X=0.30$ and the fact that mass diffusivities between the droplet and the flame would have been larger, leading to faster diffusion rates and possibly less time for pyrolysis to occur.

The flame image in Figure 2 shows that the flame is not spherical and that soot or soot related agglomerations are projected in a preferential direction. Conversely, flames in the droplet view camera were generally quite spherical. The asymmetry in the flame-view camera is likely a result of small but nonzero gravity levels in the experiments, leading to weak buoyant flows and transport of particles in a preferential direction (Figure 2).

During an experiment, droplets were allowed to stabilize between the deployment needles such that they were essentially stationary in reduced gravity before deployment occurred via needle retraction. The retraction of the needles generally caused droplet shape oscillations. The ignition sparks could also cause droplet shape oscillations. The oscillations, which are evident in plots of droplet size versus time (e.g., Figure 5(a)), quickly decayed to small levels shortly after ignition, which is consistent with computational modeling [13].

Following ignition, droplets exhibited a heat-up period characterized by a nearly constant diameter as the droplets heated (e.g., Figure 5(a)). Following this heatup period, the droplet diameter histories in all tests closely followed the $d^{2}$-law, that is, where the square of the droplet diameter decreased linearly with time. Burning rate constants, $K$, were calculated by fitting straight lines to the $d^{2}-t$ data where the initial periods associated with droplet heating were excluded. Specifically, $K$ values are defined to be the negative of the slope on a $d^{2}-t$ plot as shown in Figure 6. Burning rate constants are plotted in Figure 7 as a function of the initial droplet diameter, $d_{0}$.

The uncertainties in the measurements are estimated as follows. For the droplet size measurements, there will be errors in measurements of the droplet-gas interface locations. These errors will result from several sources, for example, 


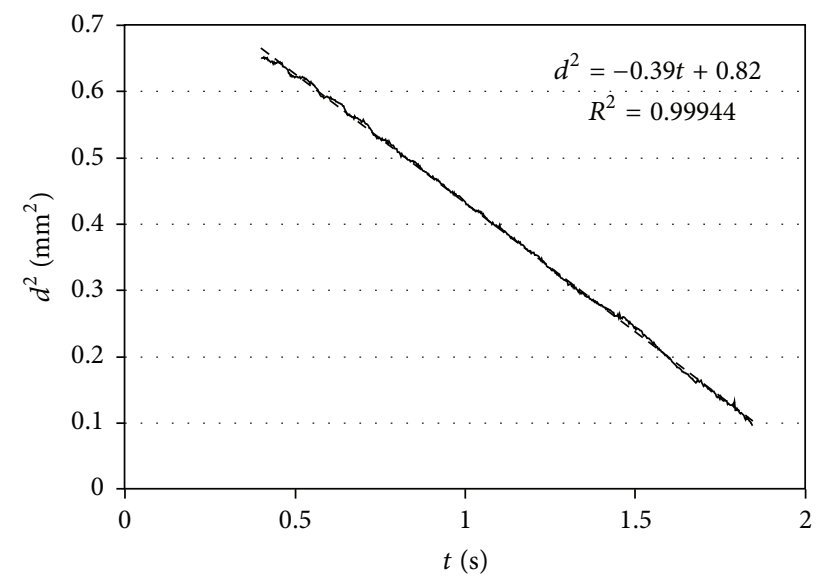

FIGURE 6: Representative plot of $d^{2}$ versus $t$ and the curve fit used to calculate the burning rate $K$ for a heptane/methanol droplet in air. The dashed line is the curve fit and the solid line is the experimental data.

the pixilation of the digital camera, calibration errors, and the fact that a cine film has finite grain sizes. In the present experiments, the resolution of the digital camera was typically about 210 pixels $/ \mathrm{mm}$; that is, a single pixel corresponded to about $5 \mu \mathrm{m}$. Film grains were typically about 4 pixels $(20 \mu \mathrm{m})$ in size and the calibration is estimated to be accurate to within about $0.01 \mathrm{~mm}(10 \mu \mathrm{m})$. Combining these errors using the root-sum-square method yields droplet size uncertainties of about $U_{d}=\left(20^{2}+5^{2}+10^{2}\right)^{1 / 2}=23 \mu \mathrm{m}(0.023 \mathrm{~mm})$, that is, $d=d_{\text {measured }} \pm 0.023 \mathrm{~mm}$. These uncertainty estimates are plotted in Figure 5(a) as the error bars.

Uncertainties in the flame size measurements are mostly determined by variations in the brightness of the fiber, which often caused blooming in the cine images (Figure 4(a)) such that the flame location could be determined only within a finite pixel range. Estimates based on the observation of the fiber glows for various experiments suggest that flame diameters can typically be determined to within about \pm $500 \mu \mathrm{m}$. These flame size uncertainties are plotted as the error bars in Figure 5(b), which shows representative flame sizes.

Because burning rates depend on the slope of a $d^{2}$ $t$ plot, relative uncertainties in burning rates $\left(U_{K} / K\right)$ are estimated as twice the relative uncertainty of the droplet size measurement, where we use $U_{d} / d_{0}$ for this estimate. The resulting uncertainties in the burning rates are shown as the error bars in Figure 7.

The addition of ethanol or methanol to a heptane droplet did not influence burning rates appreciably (Figure 7). This is likely because the initial mass fractions of ethanol or methanol were small so that burning rates were dominated by the heptane. It is also noted that in most experiments the droplets did not exhibit extinction because of the limited burning time in reduced gravity. It was only in one experiment with a heptane/methanol droplet in air that the droplet extinguished at a diameter measured to be about $250 \mu \mathrm{m}$. The extinction event was evident from the disappearance of the flame and a drastic reduction in evaporation rates.

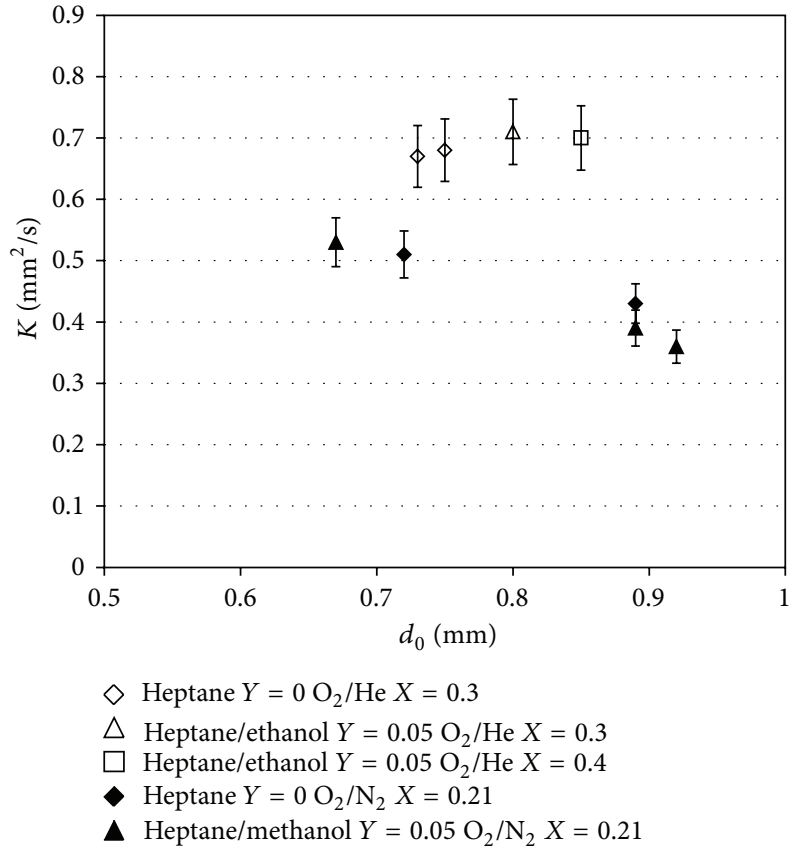

FIgURE 7: Droplet burning rates $K$ as a function of the initial droplet diameter $d_{0}$.

The data in Figure 7 show that even when uncertainties are accounted for, droplet burning rates decrease appreciably as the initial droplet diameter is increased for combustion in air. This behavior is consistent with available data for combustion of $n$-heptane droplets in air [14]. The reason for the decrease is because as droplets become larger, so do their flames. As a result, radiant heat losses from flames, which approximately scale with the cube of the flame diameter, increase appreciably, cooling the gas in the droplet vicinity. This cooling decreases conduction heat transfer to droplets, causing droplet burning rates to decrease.

Conversely, when the environment was an oxygen/helium mixture, droplet burning rates did not exhibit a significant variation with $d_{0}$ within the uncertainty of the data. This could be due to the fact that the initial droplet diameter range for these droplets was smaller than when air was used as the ambient gas. It is also noted that helium will decrease flame temperatures and temperatures of hot gas zones because of its high thermal conductivity. This could lead to smaller radiant heat losses from the hot gases surrounding a droplet than when the ambient was air, thus resulting in smaller variations in burning rates with the initial droplet diameter.

It is noted that the droplet-gas relative velocity and the presence of a support fiber can have an appreciable influence on droplet burning rates [15-17]. In the present experiments, the use of a thin tethering fiber and a symmetric ignition system kept droplet velocities very small, for example, $1 \mathrm{~mm} / \mathrm{s}$ or less, as measured from the droplet-view camera. As a result, convective effects were likely negligible. It is also noted that the use of a fiber can influence droplet combustion characteristics such as burning rates, for example, by increasing heat transfer rates into a droplet. Farouk and Dryer [17] 
performed numerical simulations of droplets that are burning in reduced gravity, both with and without support fibers. They concluded that burning rates are increased by about $1 \%$ or less if the ratio of the fiber diameter to the initial droplet diameter $\left(d_{0} / d_{\text {fiber }}\right)$ is about 42.5 or larger. Burning rates are increased by about $5 \%$ or less if $d_{0} / d_{\text {fiber }}>22$. In the present experiments, $d_{0} / d_{\text {fiber }} \in[54,67]$ so effects of the fiber on burning rates were likely small.

\section{Conclusions}

These experiments have demonstrated that burning rates of submillimeter heptane/methanol and heptane/ethanol droplets decrease with the initial droplet diameter when the ambient is air. In contrast, such decreases were not observed for the oxygen/helium ambients investigated. It was also noted that for the initial methanol or ethanol mass fractions investigated here, these alcohols did not produce significant changes in burning rates relative to pure heptane droplets. It would be of interest to perform further experiments with these mixtures, for example, covering a larger droplet diameter range than was possible in the present research. It would also be interesting to increase the alcohol mass fraction in a droplet, though there may be liquid-liquid solubility limits in the heptane/methanol system that would have to be accounted for. Modeling efforts should also be pursued, especially considering the fact that these mixtures are not ideal.

\section{Acknowledgments}

The financial support of NASA is gratefully acknowledged. The authors also thank NASA for providing the film transport system used in this research. The technical monitor was Dr. D. L. Dietrich. Gratitude is expressed to K. Hoi and C. Vang for efforts related to the development of calibration procedures and analysis of images.

\section{References}

[1] G. S. Jackson, C. T. Avedisian, and J. C. Yang, "Soot formation during combustion of unsupported methanol/toluene mixture droplets in microgravity," Proceedings of The Royal Society of London A, vol. 435, pp. 359-369, 1991.

[2] J. H. Bae and C. T. Avedisian, "Experimental study of the combustion dynamics of jet fuel droplets with additives in the absence of convection," Combustion and Flame, vol. 137, no. 1-2, pp. 148-162, 2004.

[3] C. T. Avedisian and B. J. Callahan, "Experimental study of nonane/hexanol mixture droplet combustion without natural or forced convection," Proceedings of the Combustion Institute, vol. 28, no. 1, pp. 991-997, 2000.

[4] J. Lekan, D. Gotti, A. J. Jenkins, J. C. Owens, and M. R. Johnston, "User's guide for the 2. 2 second drop tower of the NASA Lewis Research Center," NASA TM 107090, 1996.

[5] I. Aharon and B. D. Shaw, "Estimates of liquid species diffusivities from experiments on reduced-gravity combustion of heptane-hexadecane droplets," Combustion and Flame, vol. 113, no. 4, pp. 507-518, 1998.
[6] “Film Transport System Model 31101 Manual \#5058-3," Mekel Engineering, 1993.

[7] R. B. Klimek, T. W. Wright, and R. S. Sielken, "Color image processing and object tracking system," NASA TM-107144, 1996.

[8] "MATLAB Instrument Control Toolbox Documentation," The Mathworks, 2006.

[9] "MATLAB Documentation," The Mathworks, 2006.

[10] "MATLAB Image Processing Toolbox Documentation," The Mathworks, 2006.

[11] N. Otsu, "A threshold selection method from gray-level histograms," IEEE Transactions on Systems, Man, and Cybernetics, vol. 9, no. 1, pp. 62-66, 1979.

[12] M. Y. Choi, F. L. Dryer, J. B. Haggard Jr., and M. H. Brace, "Further observations on microgravity droplet combustion in the NASA-Lewis drop tower facilities: a digital image processing technique for droplet burning data," AIP Conference Proceedings, vol. 197, pp. 338-361, 1990.

[13] J. Yan and B. D. Shaw, "Numerical simulation of unsteady flows and shape oscillations in liquid droplets induced by deployment needle retraction," Microgravity Science and Technology, vol. 22, no. 1, pp. 17-26, 2010.

[14] G. S. Jackson and C. T. Avedisian, "Effect of initial diameter in spherically symmetric droplet combustion of sooting fuels," Proceedings of The Royal Society of London A, vol. 446, no. 1927, pp. 255-276, 1994.

[15] M. Y. Choi, F. L. Dryer, and J. B. Haggard Jr., "Observations on a slow burning regime for hydrocarbon droplets: n-Heptane/air results," Symposium (International) on Combustion, vol. 23, no. 1, pp. 1597-1604, 1991.

[16] M. Ackerman and F. A. Williams, "Simplified model for droplet combustion in a slow convective flow," Combustion and Flame, vol. 143, no. 4, pp. 599-612, 2005.

[17] T. Farouk and F. L. Dryer, "Microgravity droplet combustion: effect of tethering fiber on burning rate and flame structure," Combustion Theory and Modelling, vol. 15, no. 4, pp. 487-515, 2011. 

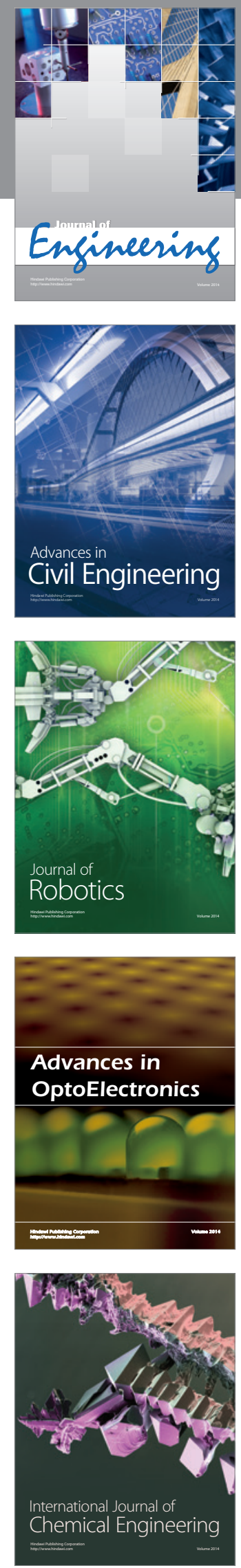

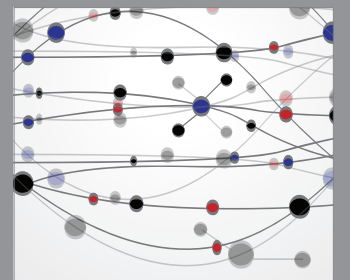

The Scientific World Journal
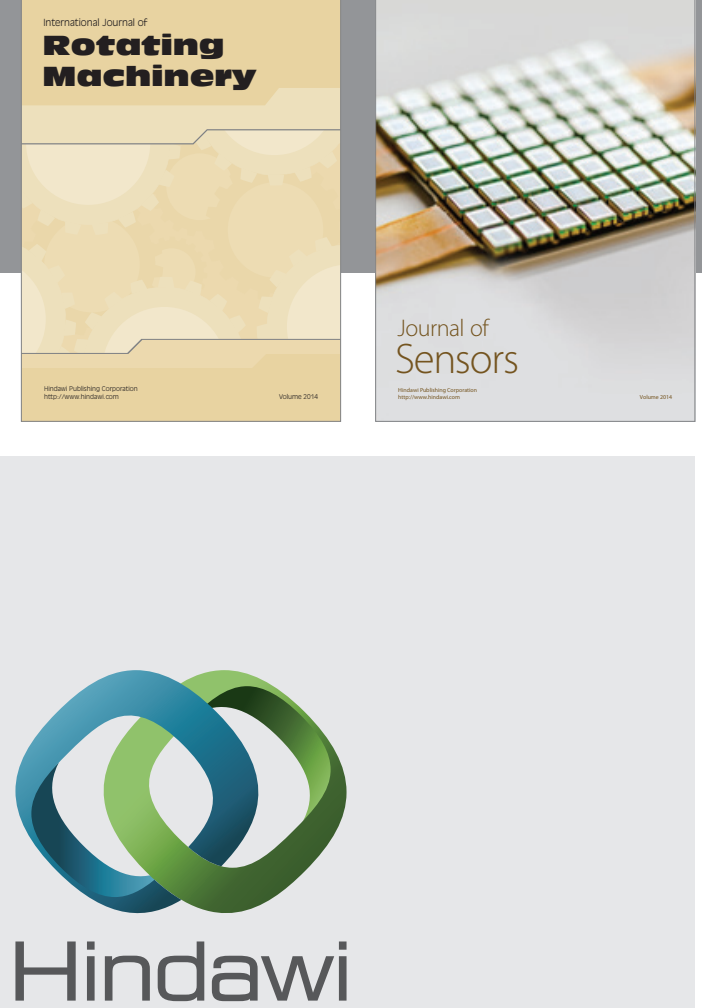

Submit your manuscripts at http://www.hindawi.com
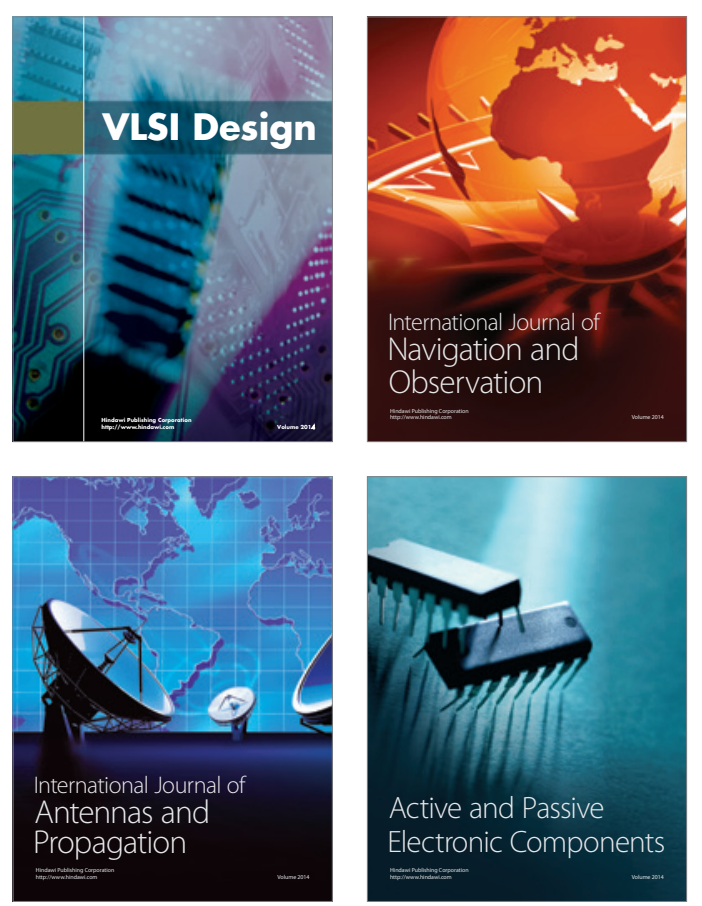
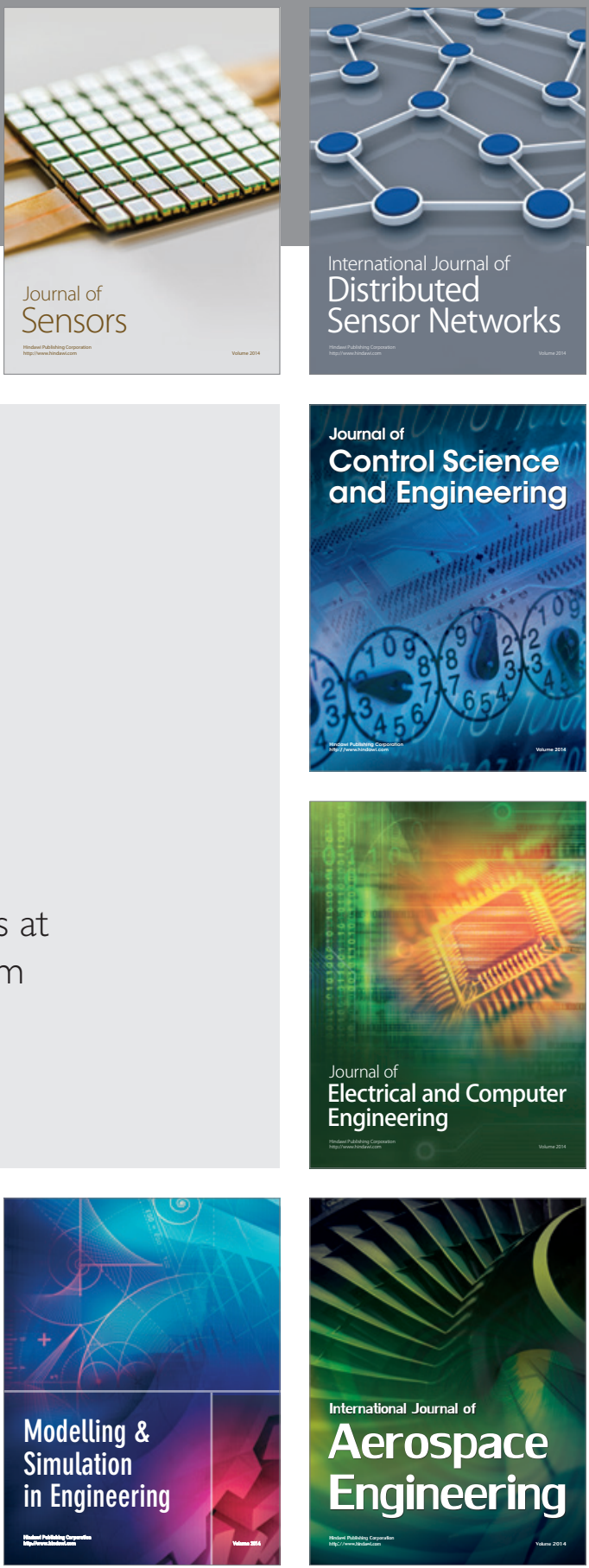

Journal of

Control Science

and Engineering
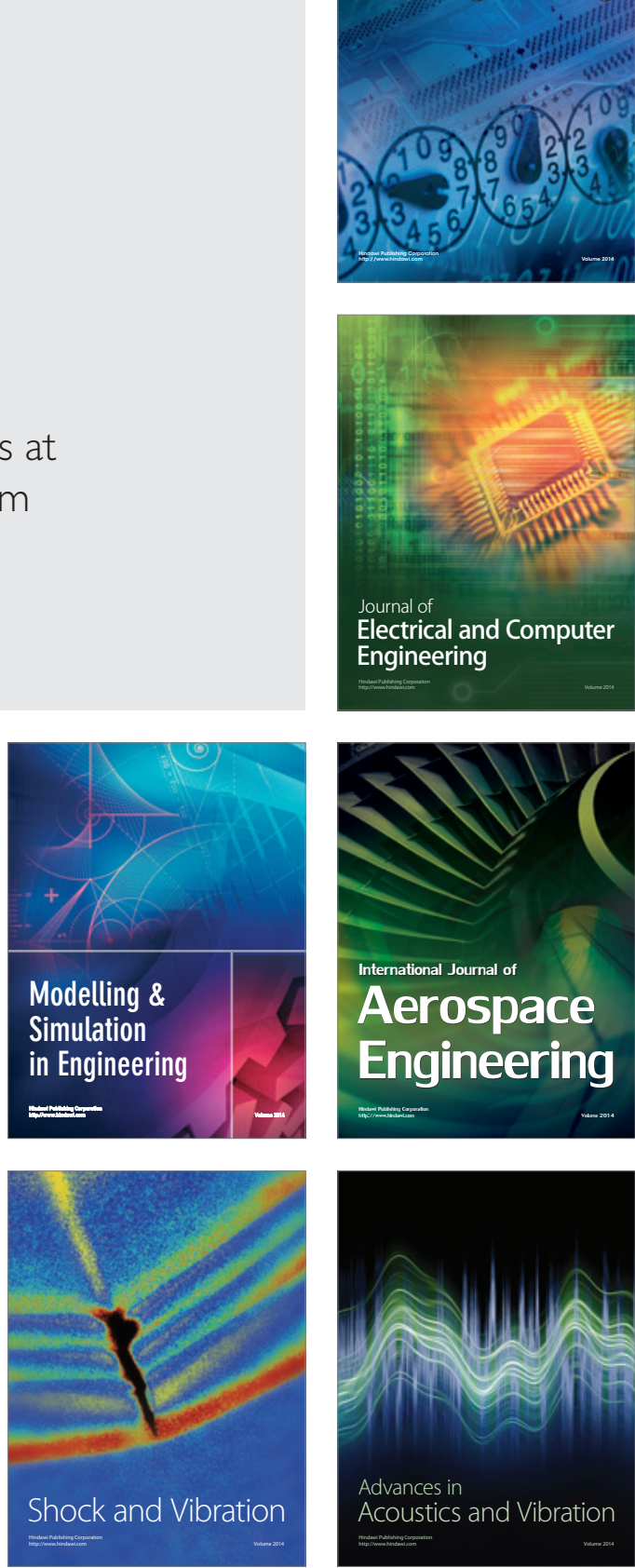